PROCEEDINGS OF THE

AMERICAN MATHEMATICAL SOCIETY

Volume 125, Number 1, January 1997, Pages 183-187

S 0002-9939(97)03552-1

\title{
SOME RELATIONS BETWEEN NÖRLUND AND ABEL SUMMABILITY
}

\author{
J. R. NURCOMBE
}

(Communicated by Theodore Gamelin)

\begin{abstract}
The definition of generalised Abel summability is extended to positive orders and a definition of strong generalised Abel summability is introduced. A result of Jurkat and Peyerimhoff concerning the implication between Nörlund and generalised Abel summabilities is extended to positive integral order. Analogous results for the corresponding strong methods are also given.
\end{abstract}

\section{INTRODUCTION}

Let $(N, p)$ denote the Nörlund method of summability of a sequence $\left(s_{n}\right)$ of real or complex numbers, and $\left(A_{0}^{*}\right)$ the generalised Abel summability method. Concerning the implication between these methods of summability, Jurkat and Peyerimhoff proved the following result [5, Lemma 1]:

Theorem A. If $(N, p)$ is real and regular, and $s_{n} \rightarrow s(N, p)$, then $s_{n} \rightarrow s\left(A_{0}^{*}\right)$.

In the case when $p_{0}>0, p_{n} \geq 0,(N, p)$ is regular, Zygmund and, independently, Silverman and Tamarkin, proved that $s_{n} \rightarrow s(N, p)$ implies $s_{n} \rightarrow s\left(A_{0}^{*}\right)$, where $\left(A_{0}^{*}\right)$ is a slightly more restrictive method than that considered in Theorem A (see Hardy's book [4, Theorem 18] for details).

In a recent note [8], Sarigöl considered the same question. His result is incorrect however, because he assumed that the quotient of two power series each with unit radius of convergence is a power series also having unit radius of convergence. This error has appeared before in related circumstances (see for example [7], where results for absolute summability, analogous to those considered here, are obtained).

The object of this note is to extend Theorem A so as to obtain conditions for which $(N, p)$ summability implies $\left(A_{\mu}^{*}\right)$ summability, where $\mu$ is a positive integer (see $\S 2$ below, for relevant definitions). The results enable deduction of implications between the corresponding strong methods of summability.

This work is based on part of the author's M.Sc. thesis written at the University of Birmingham in 1978, under the supervision of Dr. B. Thorpe and the late Professor B. Kuttner. Their advice and encouragement are acknowledged with gratitude.

Received by the editors March 9, 1995 and, in revised form, July 13, 1995

1991 Mathematics Subject Classification. Primary 40E05, 40F05, 40 G05.

Key words and phrases. Summability, strong, Nörlund, Abel, convergence, sequence.

(c)1997 American Mathematical Society 


\section{NotATION AND DEFINITIONS}

Denote by $\left(p_{n}\right)$ a sequence of real numbers, and write $P_{n}=\sum_{r=0}^{n} p_{r}, P_{n}^{(1)}=$ $\sum_{r=0}^{n} P_{r}$, etc. If

$$
\frac{\sum_{v=0}^{n} p_{n-v} s_{v}}{P_{n}} \rightarrow s \quad \text { as } n \rightarrow \infty,
$$

then the sequence $\left(s_{n}\right)$ is said to be Nörlund summable to $s$, and we write $s_{n} \rightarrow$ $s(N, p)$. In order for the $(N, p)$ method to be regular, that is, for $s_{n} \rightarrow s$ to imply $s_{n} \rightarrow s(N, p)$, it is necessary and sufficient that $p_{n}=o\left(P_{n}\right)$ and $\sum_{v=0}^{n}\left|p_{v}\right|=$ $O\left(\left|P_{n}\right|\right)$. If

$$
\sum_{n=0}^{N}\left|p_{n}\right|\left|\frac{\sum_{v=0}^{n} p_{n-v} a_{v}}{p_{n}}-s\right|^{\lambda}=o\left(P_{N}\right)
$$

then $\left(s_{n}\right)$ is said to be strongly Nörlund summable to $s$ with index $\lambda>0$, and we write $s_{n} \rightarrow s[N, p]_{\lambda}$ (see [2]). In what follows, $\sum s_{n} x^{n}$ denotes $\sum_{n=0}^{\infty} s_{n} x^{n}$, etc.

A sequence $\left(s_{n}\right)$ is said to be generalised Abel summable of order $\mu$ to $s$, or briefly $\left(A_{\mu}^{*}\right)$ summable to $s$, and we write $s_{n} \rightarrow s\left(A_{\mu}^{*}\right), \mu>-1$, if $(1-x)^{\mu+1} \sum\left(\begin{array}{c}n+\mu \\ n\end{array}\right) s_{n} x^{n}$ has a positive radius of convergence and defines an analytic function $\sigma_{\mu}(x)$, regular in the unit disc except for poles, none of which lies on the interval $1-\varepsilon<x<1$ for an arbitrarily small fixed positive $\varepsilon$, and $\sigma_{\mu}(x) \rightarrow s$ when $x \rightarrow 1$-through real values. This definition is based on the $\left(A_{\mu}\right)$ method of Borwein [1]. The proof of Theorem A in [5] involves a similar definition to $\left(A_{\mu}^{*}\right)$ when $\mu=0$. There, poles are allowed anywhere in the unit disc but regularity is restricted to $1-\varepsilon<x<1$. However, as the proof makes clear, poles cannot occur on $1-\varepsilon<x<1$, so that the $\left(A_{0}^{*}\right)$ method in [5] implies our $\left(A_{0}^{*}\right)$ method, when considering implications between $(N, p)$ and $\left(A^{*}\right)$. A slightly more restrictive definition than $\left(A_{\mu}^{*}\right)$ above, excludes poles from the interval $0 \leq x<1$, but is otherwise identical. The case $\mu=0$ is that alluded to after Theorem A in $\S 1$.

In order to define strong generalised Abel summability $\left[A_{\mu}^{*}\right]_{\lambda}, \lambda \geq 1$, it is convenient to make a change of variable by writing $x=\frac{y}{1+y}$. Now if $s_{n} \rightarrow s\left(A_{\mu}^{*}\right)$ and $\int_{M}^{Y}\left|y \sigma^{\prime}(y)\right|^{\lambda} d y=o(Y)$ as $Y \rightarrow \infty$, then $\left(s_{n}\right)$ is strongly generalised Abel summable of order $\mu$ with index $\lambda$ to the sum $s$, or $s_{n} \rightarrow s\left[A_{\mu}^{*}\right]_{\lambda}$. Here, $M$ is an arbitrarily large fixed positive number and $\sigma^{\prime}(y)$ denotes the derivative of $\sigma(y)$ with respect to $y$, where $\sigma(y)=\sigma_{\mu}\left(\frac{y}{1+y}\right)$. This definition is based on the $\left[A_{\mu-1}\right]_{\lambda}$ method of Mishra $\left[6\right.$, Theorem 4]. The more restrictive definition of $\left[A_{\mu}^{*}\right]_{\lambda}$, where poles are excluded from $0 \leq x<1$ in $\left(A_{\mu}^{*}\right)$, is obtained by replacing the lower limit of integration $M$ by zero, and using the appropriate definition of $\left(A_{\mu}^{*}\right)$.

\section{RESUlts AND PROOFS}

Theorem 1. Let $(N, p)$ be real and regular, and $n\left|p_{n}\right|=O\left(\left|P_{n}\right|\right)$. Then $s_{n} \rightarrow$ $s(N, p)$ implies $s_{n} \rightarrow s\left(A_{1}^{*}\right)$.

For the proof, we require two lemmas.

Lemma 1. Let $p_{n} \geq 0$ and $n p_{n}=O\left(P_{n}\right)$. Then $n P_{n}=O\left(P_{n}^{(1)}\right)$. 
Proof. Since $n p_{n} \leq M P_{n}$, it follows that

$$
M P_{n}^{(1)} \geq \sum_{r=0}^{n} r p_{r}=n P_{n}-\sum_{r=0}^{n-1} P_{r} \geq n P_{n}-P_{n}^{(1)}
$$

from which the conclusion follows. (I am grateful to the referee for pointing out this proof, which is simpler than my original.)

Lemma 2. Let $\left(p_{n}\right)$ be a sequence of real numbers with $(N, p)$ real and regular, and $n\left|p_{n}\right|=O\left(\left|P_{n}\right|\right)$. Then $n\left|P_{n}\right|=O\left(\left|P_{n}^{(1)}\right|\right)$.

Proof. Since $(N, p)$ is regular, $p_{n}=o\left(P_{n}\right)$ and hence $\frac{P_{n-1}}{P_{n}} \rightarrow 1$. Thus $\left(P_{n}\right)$ is a sequence of ultimately one-signed terms, and, without loss of generality, we may take this sign as positive. Also, since $\sum_{r=0}^{n}\left|p_{r}\right|=O\left(\left|P_{n}\right|\right)$, then $P_{n}^{(1)} \rightarrow \infty$. Write $P_{n}^{(1)^{*}}=\sum_{r=0}^{n}\left|P_{r}\right|, P_{n}^{*}=\sum_{r=0}^{n}\left|p_{r}\right|$. Then $n\left|p_{n}\right|=O\left(\left|P_{n}\right|\right)$ implies $n\left|p_{n}\right|=O\left(P_{n}^{*}\right)$ which implies $n P_{n}^{*}=O\left(P_{n}^{(1)^{*}}\right)$, by Lemma 1. Since $P_{n}^{(1)} \rightarrow \infty$ and only a finite number of the $P_{n}$ are negative, $P_{n}^{(1)^{*}}=O\left(P_{n}^{(1)}\right)$ so that $n\left|P_{n}\right| \leq n P_{n}^{*}=O\left(P_{n}^{(1)}\right)$.

Proof of Theorem 1. Suppose $s_{n} \rightarrow s(N, p)$. Then $\sum(n+1) s_{n} x^{n}$ has a positive radius of convergence and, inside the circle of convergence,

$$
\begin{aligned}
(1-x)^{2} \sum(n+1) s_{n} x^{n} & =(1-x) \sum\left\{(n+1) s_{n}-n s_{n-1}\right\} x^{n} \\
& =(1-x) \sum s_{n} x^{n}+(1-x) \sum n a_{n} x^{n} .
\end{aligned}
$$

By Theorem A, $s_{n} \rightarrow s\left(A_{0}^{*}\right)$, so that it suffices to prove $n a_{n} \rightarrow 0\left(A_{0}^{*}\right)$. Writing $a(x)=\sum a_{n} x^{n}=\frac{T(x)}{P(x)}$, where $T(x)=\sum P_{n} t_{n} x^{n}$, and $t_{n}$ is the $(N, p)$ transform of $\left(s_{n}\right)$, we have, inside the circle of convergence,

$$
(1-x) \sum n a_{n} x^{n}=(1-x) x a^{\prime}(x)=(1-x) x\left\{\frac{T^{\prime}(x)}{P(x)}-\frac{T(x) P^{\prime}(x)}{[P(x)]^{2}}\right\} ;
$$

the primes denote differentiation with respect to $x$. The last expression equals

$$
\begin{aligned}
(1-x) & \left\{\frac{\sum n P_{n} t_{n} x^{n}}{P(x)}-\frac{\sum P_{n} t_{n} x^{n} \sum n P_{n} x^{n}}{[P(x)]^{2}}\right\} \\
= & \frac{1}{\sum P_{n}^{(1)} x^{n}}\left\{\sum n P_{n} t_{n} x^{n}-\sum P_{n} t_{n} x^{n} \frac{\sum n P_{n} x^{n}}{P(x)}\right\} \\
= & \frac{1}{\sum P_{n}^{(1)} x^{n}} \sum\left\{n P_{n} x^{n}-\frac{P_{n} x^{n}}{P(x)} \sum n P_{n} x^{n}\right\} t_{n} \equiv \sum c_{n}(x) t_{n}
\end{aligned}
$$

say, where

$$
c_{n}(x)=\frac{n P_{n} x^{n}-P_{n} x^{n} \sum n P_{n} x^{n} / P(x)}{\sum P_{n}^{(1)} x^{n}} .
$$

We wish to show that when $t_{n} \rightarrow s, \sum c_{n}(x) t_{n} \rightarrow 0$ as $x \rightarrow 1-$. This is a sequence to function transformation, and using a suitable modification of Theorem 5 in [4], the necessary and sufficient conditions for the desired property to hold are (i) $\sum\left|c_{n}(x)\right|<H$, where $H$ is positive constant independent of $x$ for $0<x_{0} \leq x<1$, (ii) $\sum c_{n}(x) \rightarrow 0$ as $x \rightarrow 1-$, and (iii) $c_{n}(x) \rightarrow 0$ as $x \rightarrow 1-$, for each $n$. 
For (i), we require

$$
\sum\left|c_{n}(x)\right| \leq \frac{\sum n\left|P_{n}\right| x^{n}}{\left|\sum P_{n}^{(1)} x^{n}\right|}+\frac{\sum\left|P_{n}\right| x^{n}\left|\sum n P_{n} x^{n}\right|}{\left|\sum P_{n} x^{n}\right|\left|\sum P_{n}^{(1)} x^{n}\right|} \leq H
$$

when $0<x_{0} \leq x<1$. As in the proof of Lemma $2,\left(P_{n}\right)$ must be ultimately one-signed, and we can take this sign as positive. Thus $\left(P_{n}^{(1)}\right)$ is also ultimately positive, and since $P_{n}^{(1)} \rightarrow \infty$, the contribution of the negative terms to the sums will not affect the boundedness or otherwise of (3.2) for values of $x$ sufficiently near 1. Thus the modulus signs in (3.2) can be dropped, and (3.2) holds provided $n P_{n}=O\left(P_{n}^{(1)}\right)$, which follows from Lemma 2. Plainly, (ii) holds, since $\sum c_{n}(x)$ vanishes identically in this case. For (iii), we have from (3.1) that, for each $n$, $\lim _{x \rightarrow 1-} n P_{n} x^{n}=n P_{n}, \lim _{x \rightarrow 1-} P_{n} x^{n}=P_{n}$ are both finite, but both $\sum P_{n}^{(1)} x^{n}$ and $P(x)$ tend to infinity as $x \rightarrow 1-$. Finally,

$$
\sup _{0 \leq x<1} \frac{\left|\sum n P_{n} x^{n}\right|}{\sum P_{n}^{(1)} x^{n}}<K
$$

a positive constant, provided $n P_{n}=O\left(P_{n}^{(1)}\right)$, by the same argument as in (i). This completes the proof.

Remarks. 1. The reader should have no difficulty in formulating and proving the analogous result when $\left(p_{n}\right)$ is positive and $\left(A_{1}^{*}\right)$ refers to the method in which poles are excluded from $0 \leq x<1$. We shall take such modifications for granted from now on.

2. With the same hypotheses, Theorem 1 can be extended to the case when $(N, p)$ implies $\left(A_{\mu}^{*}\right)$ where $\mu$ is a positive integer. The proof involving induction on $\mu$ follows the same lines as that of Theorem 1 , but requires $\mu$ differentiations of the quotient $\frac{T(x)}{P(x)}$. The details, while straightforward in principle, are somewhat involved in practice, and are consequently omitted.

3. The general case, when $\mu$ is a positive (non-integral) number, involves the difficulty that while $\left(A_{\lambda}\right) \Rightarrow\left(A_{\mu}\right)$ for $\lambda>\mu>-1[1$, Theorem 2], it is not necessarily true that $\left(A_{\lambda}^{*}\right) \Rightarrow\left(A_{\mu}^{*}\right)$. The problem is that while $s_{n} \rightarrow s\left(A_{\lambda}^{*}\right)$, the function $\sigma_{\mu}(x)$ can have branch points in the unit circle, so that the definition of $\left(A_{\mu}^{*}\right)$ no longer holds. I have not been able to solve this problem, and suggest that if the implication can be proved at all, it will require a different method of proof from the above.

4. To obtain relations between strong Nörlund and strong Abel summability, we apply the analogue of an argument given by Flett [3, Theorem 4(i)] and consider the sequence of implications: $[N, p]_{\lambda} \Rightarrow(N, p) \Rightarrow\left(A_{\mu}^{*}\right) \Rightarrow\left[A_{\mu}^{*}\right]_{\lambda}$, where the $\lambda$ 's $(\geq 1)$, need not be equal.

The first implication holds under conditions given in [2, Theorems 6 and 7], the second is considered above, and the third follows from the relation

$$
y \sigma_{\mu}^{\prime}(y)=(\mu+1)\left[\sigma_{\mu+1}(y)-\sigma_{\mu}(y)\right]
$$

and regularity of the $(C, 1)$ mean for integrals. The result obtained is summarised in.

Theorem 2. Let $(N, p)$ be real and regular with $n\left|p_{n}\right|=O\left(\left|P_{n}\right|\right)$. Then $s_{n} \rightarrow$ $s[N, p]_{\lambda}$ implies $s_{n} \rightarrow s\left[A_{\mu}^{*}\right]_{\lambda}, \lambda \geq 1, \mu$ a non-negative integer. 


\section{REFERENCES}

1. D. Borwein, On a scale of Abel type summability methods, Proceedings Cambridge Phil. Soc. 53 (1957), 318-322. MR 19:134f

2. D. Borwein and F. P. Cass, Strong Nörlund summability, Math. Zeitschrift 103 (1968), 94111. MR 37:643

3. T. M. Flett, Some remarks on strong summability, Quart. Journ. of Math. (Oxford) 10 (1959) 115-139. MR 21:6491

4. G. H. Hardy, Divergent Series, (Oxford 1949). MR 11:25a

5. W. Jurkat and A. Peyerimhoff, The consistency of Nörlund and Hausdorff methods, Annals of Math. 62 (1955), 498-503. MR 17:359a

6. B. P. Mishra, Strong summability of infinite series on a scale of Abel type summability methods, Proceedings Cambridge Phil. Soc. 63 (1967), 119-127. MR 34:3156

7. J. R. Nurcombe, On absolute Nörlund and Abel summability, Journ. Indian Math. Soc. 53 (1988), 217-226. MR 90k:40008

8. M. A. Sarigöl, A relation between Nörlund and Abel summabilities, Proceedings American Math. Soc. 122 (1994), 223-225. MR 95d:40015

South Birmingham College, Cole Bank Road, Hall Green, Birmingham B28 8ES, EngLAND 